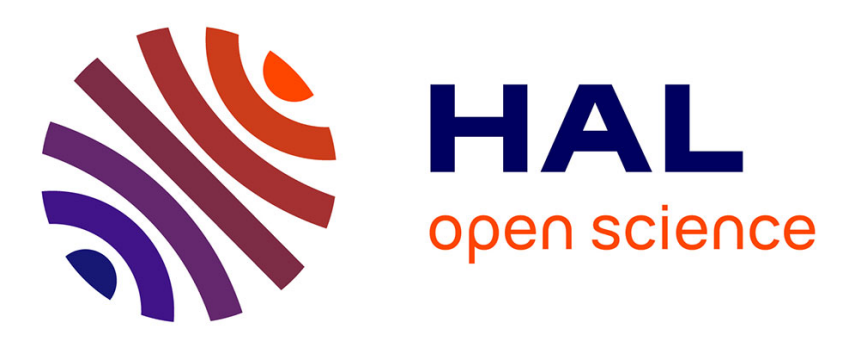

\title{
Hole growth dynamics in a two dimensional Leidenfrost droplet
}

Christophe Raufaste, Franck Celestini, Alexandre Barzyk, Thomas Frisch

\section{To cite this version:}

Christophe Raufaste, Franck Celestini, Alexandre Barzyk, Thomas Frisch. Hole growth dynamics in a two dimensional Leidenfrost droplet. Physics of Fluids, 2015, 27 (3), pp.031704. 10.1063/1.4916622.1. hal-01211360

\section{HAL Id: hal-01211360 https://hal.science/hal-01211360}

Submitted on 5 Oct 2015

HAL is a multi-disciplinary open access archive for the deposit and dissemination of scientific research documents, whether they are published or not. The documents may come from teaching and research institutions in France or abroad, or from public or private research centers.
L'archive ouverte pluridisciplinaire HAL, est destinée au dépôt et à la diffusion de documents scientifiques de niveau recherche, publiés ou non, émanant des établissements d'enseignement et de recherche français ou étrangers, des laboratoires publics ou privés. 


\section{Hole growth dynamics in a two dimensional Leidenfrost droplet}

Christophe Raufaste, ${ }^{1}$ Franck Celestini, ${ }^{1}$ Alexandre Barzyk, ${ }^{1}$ and Thomas Frisch ${ }^{2}$

1) Université Nice Sophia Antipolis, CNRS, LPMC - UMR 7336, Parc Valrose, 06100 Nice, France

${ }^{2)}$ Université Nice Sophia Antipolis, CNRS, INLN, UMR 7335, 1361 Routes des lucioles, Sophia Antipolis F-06560 Valbonne, France

We studied the behaviors of Leidenfrost droplets confined in a Hele-Shaw cell. These droplets are unstable above a critical size and a hole grows at their center. We experimentally investigate two different systems for which the hole growth dynamics exhibits peculiar features that are driven by capillarity and inertia. We report a first regime characterized by the liquid reorganization from a liquid sheet to a liquid torus with similarities to the burst of micron-thick soap films. In the second regime the liquid torus expands and thins before fragmentation. Finally we propose models to account for the experimental results.

PACS numbers: 66.30.Qa, 05.70.Ln, 81.15.Aa

In spite of its discovery in the eighteen century ${ }^{1}$, the Leidenfrost phenomenom is still today the subject of intensive research. Free standing Leidenfrost droplets represent a model system of absolute super-hydrophobicity and have been intensively described both theoretically and experimentally in the previous years ${ }^{2,3}$. The dynamics of these droplets are displaying evidences of numerous fundamental phenomena in drop dynamics and more generally in capillary fluid dynamics $^{4}$. Applications are futhermore numerous among the control of the cooling of metals in metallurgy ${ }^{5}$, the nuclear reactor safety ${ }^{6}$, self-propulsion for transport properties ${ }^{7}$ and micro-droplets evaporation ${ }^{8}$. Several studies are actually also devoted to the possible control of these droplets under the action of different kind of external forces ${ }^{9,10}$.

We have recently shown ${ }^{11}$ that Leidenfrost droplets in a confined Hele-Shaw geometry exhibit two new phenomena: an intriguing morphogenic instability and a spontaneous hole nucleation and growth above a critical size. This latter point is the subject of this study. In this manuscript we investigate the hole growth dynamics in two dimensional Leidenfrost droplets. To summarize, we first put in evidence a twofold growth behavior. In the first regime we show that the hole growth is driven by a capillary-inertial mechanism at the millimeter scale that is similar to the hole growth in micron-thick liquid sheets such as soap films that has been rationalized by the seminal work of Culick ${ }^{12}$. The radius of the hole expands with a power law and an exponent smaller than unity. We report that the Leidenfrotst system exhibits the properties of recessing liquid sheets of variable thickness as described by Keller ${ }^{13}$. To our knowledge such a case was never observed experimentally. The second regime is characterized by a different growth law in which the liquid expands outward at constant speed. In both regimes, the friction forces are negligible and the capillary and the inertia are the only driving forces.

The experimental setup has been previously described in Celestini et al. ${ }^{11}$. A liquid droplet is confined between two horizontal plates separated by a spacing $d$. The plates temperature is controlled and set to values largely higher than the Leidenfrost temperature. As a consequence, the presence of insulating vapor layers on both faces avoid any direct contacts between the droplet and the surfaces. Two systems are considered:

(1) liquid water droplets confined between a copper and a sapphire plates that are set to a temperature of $300^{\circ} \mathrm{C}$. The drop temperature is assumed to be close to its boiling temperature and therefore nearly equal to $100^{\circ} \mathrm{C}$. At this temperature, the density and the surface tension of the liquid water are respectively $\rho_{w}=958 \mathrm{~kg} \cdot \mathrm{m}^{-3}$ and $\gamma_{w}=0.059$ $\mathrm{kg} \cdot \mathrm{s}^{-2}$.

(2) liquid nitrogen droplets that are placed between two glass plates at room temperature. Nitrogen droplets are evaporating at $-196^{\circ} \mathrm{C}$. At this temperature, the density and the surface tension of the liquid equal respectively $\rho_{N_{2}}=808 \mathrm{~kg} \cdot \mathrm{m}^{-3}$ and $\gamma_{N_{2}}=0.0085 \mathrm{~kg} \cdot \mathrm{s}^{-2}$.

Droplets are grown slowly by coalescence with smaller droplets projected continuously. It is worth noticing that producing such a continuous flow of small nitrogen droplets is not an easy task. A special apparatus has therefore been constructed for this purpose. Once the droplet reaches a maximal radius $R_{0}$, it becomes unstable and a hole nucleates at its center and grows. As we observed and explained ${ }^{11}, R_{0}$ versus $d$ measurements exhibit a quasi-linear trend with some fluctuations of the $R_{0}$ values for a given $d$, mostly due the fluctuating nature of the vapor layers thickness between the droplet and the plates. Averages lead to $<R_{0} / d>_{w}=5.8 \pm 1.3$ for water droplets $<R_{0} / d>_{N_{2}}=4.6 \pm 0.9$ for nitrogen.

We give in Fig. 1 an image sequence of the water droplet that is representative of the hole nucleation and growth. Images are recorded using an high speed camera at frame rates ranging from 2000 to 20000 frames per second. Image analysis permits to measure the external and internal hole projected areas, respectively written $A_{e}$ and $A_{i}$. The hole is relatively well described by a circle so that we can calculate the hole internal and external radii using respectively $R_{i}=\sqrt{A_{i} / \pi}$ and $R_{e}=\sqrt{A_{e} / \pi}$. The equivalent liquid radius can be defined from the projected area of the liquid and reads $R_{\ell}(t)=\sqrt{R_{e}(t)^{2}-R_{i}(t)^{2}}$. 

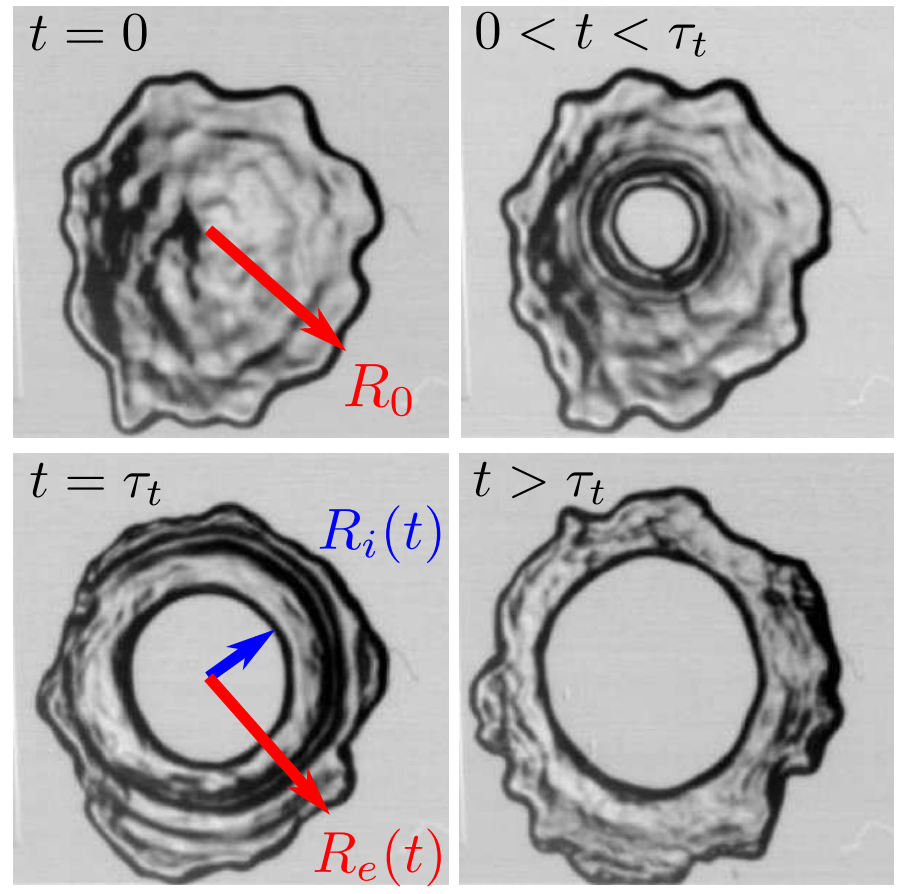

FIG. 1. Image sequence of the hole nucleation and growth in a Leidenfrost droplet of water. The plates temperature is $300^{\circ} \mathrm{C}$. Radii definition: droplet external radius $R_{e}$ and hole internal radius $R_{i}$. The length scale is given by $R_{0}=6 \mathrm{~mm}$ and the time scale is given by $\tau_{t}=6 \mathrm{~ms}$.

We represent in Fig. 2 a typical plot of the time evolution of the different radii characterizing a water Leidenfrost droplet. As indicated above, the initial radius of the droplet before the hole nucleation is noted $R_{0}=R_{e}(t=0)$. One clearly see on this plot that the dynamics is twofold with a transition time denoted $\tau_{t}$ and a corresponding hole radius $R_{t}=R_{i}\left(t=\tau_{t}\right)$.

For $t<\tau_{t}\left(R_{i}<R_{t}\right), R_{e}$ is almost constant and the equivalent liquid radius $R_{\ell}(t)$ decreases slightly. For this initial growth regime the temporal evolution of the inner radius can be fitted by a power law $R_{i}(t) \propto t^{n}$. Several experiments are performed for the two systems considered in this study. Statistics on the growth exponent $n$ leads to $\langle n\rangle_{w}=0.65 \pm 0.12$ for water and $\langle n\rangle_{N_{2}}=0.60 \pm 0.11$ for nitrogen.

For $t>\tau_{t}$, both $R_{e}$ and $R_{i}$ increase with time. The liquid equivalent radius remains constant. For this second growth regime, the increase of $R_{i}(t)$ is linear and characterized experimentally by a constant velocity $V_{2}$. There is no visible correlation between $V_{2}$ and $R_{0}$ for a given spacing $d$. We observed that this velocity decreases significantly when increasing $d$. This point will discuss below.

We now present a model for the time evolution of the radius of the droplet, that takes into account the most salient physical aspect of our system. Let us consider a gas pocket entrapped between the droplet and each plate as shown in Fig. 3. At the onset of the hole nucleation, the droplet radius equals $R_{0}$ and the liquid sheet separating the two gas pockets is very thin in its middle ${ }^{11}$. We therefore assume a linear profile $e(r)$ for the thin liquid film thickness with a zero value in its center (Fig. 3). The radius of the gas pocket at the onset of the first growth regime is denoted $R_{g}$. Our model assumes that the first growth regime corresponds to a liquid reorganization in the confining dimension without change of the external dimension. This hypothesis is confirmed in Figure 2: indeed $R_{e}$ remains constant, while the liquid equivalent radius $R_{\ell}$ decreases. At the end of the first grow regime, the gas is entrapped in the middle of the droplet which has a torus like shape. From simple geometrical considerations the gas volume entrapped before the hole nucleation reads:

$$
V_{g a z}=\iiint r d r d \theta d z=\int_{0}^{R_{g}} 2 \pi(d-e(r)) r d r=\frac{\pi}{3} d R_{g}^{2},
$$

where the triple integral is calculated on the gas domain in a cylindrical geometry $(r, \theta, z)$. At the end of the first regime, the gas is found in a cylinder of radius $R_{t}$ and $V_{g a z}=\pi d R_{t}^{2}$. Since, on this time scale, the production of vapor through evaporation is negligible, mass conservation of the gas therefore leads to :

$$
R_{g}=\sqrt{3} R_{t}
$$




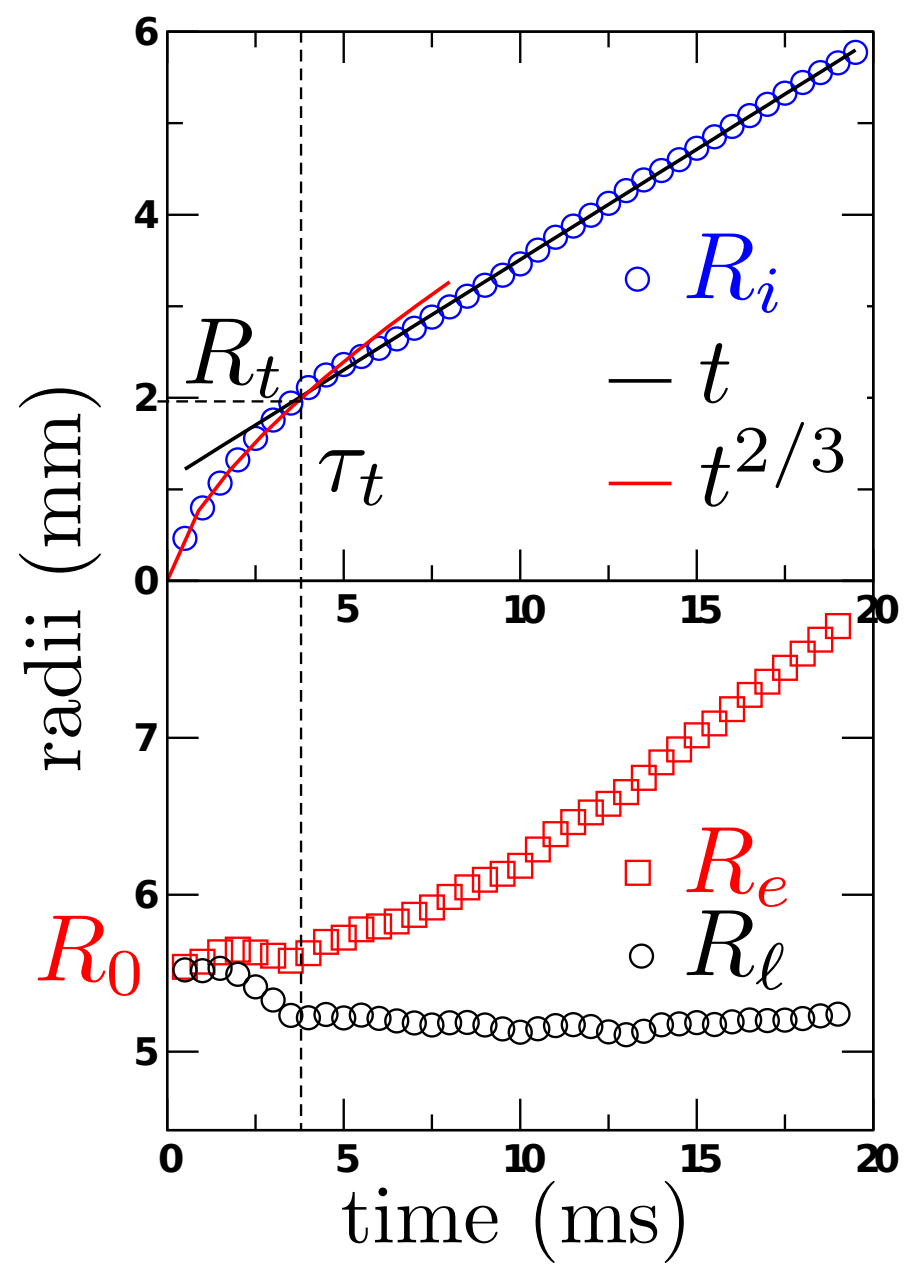

FIG. 2. Radii vs time for a typical experiment. Respectively the external $\left(R_{e}(t)\right)$, the internal $\left(R_{i}(t)\right)$, and the equivalent liquid $\left(R_{\ell}(t)\right)$ radii respectively.

The following model is derived from Culick approach and applies to the dynamics of recessing soap films of constant thickness $^{12}$. In its study, Culick assumed that all the mass of the recessing film is concentrated inside a single liquid rim that does not perturb the soap film profile upstream and that the dynamics is driven by the momentum balance equation. Following Culick approach, Keller derives with the same hypotheses the equation of a recessing liquid film of variable thickness $e(r)$ in the cylindrical geometry ${ }^{13}$ :

$$
\begin{gathered}
\frac{d}{d t}\left(\rho\left(\int_{0}^{R_{i}(t)} 2 \pi r e(r) d r\right) \frac{d R_{i}(t)}{d t}\right)= \\
2 \pi R_{i}(t) .2 \gamma\left(1+\frac{1}{4}\left(\frac{d e}{d r}\left(R_{i}(t)\right)\right)^{2}\right)^{-1 / 2},
\end{gathered}
$$

where the left-hand side of the equation is related to the momentum of the rim which concentrates all the recessing liquid. It accounts for the increase of the recessing mass as the hole opens. The liquid rim is pulled by capillary forces imposed by the gas-liquid interfaces of the upstream profile (right-hand side of the equation). For the case of an uniform sheet of thickness $e_{0}$ the result of Culick ${ }^{12}$ is retrieved and $R_{i}(t)=\left(\frac{2 \gamma}{\rho e_{0}}\right)^{1 / 2} t$. For the case of liquid films of heterogenous thickness $e(r)$, Keller looked for power law cases $e(r)=b_{a} r^{a}$. Assuming a small variation hypothesis, $\frac{d e}{d r}\left(R_{i}(t)\right)<<1$, he showed that

$$
R_{i}(t)=\left(\frac{\gamma(a+2)^{3}}{\rho b_{a}(a+4)}\right)^{1 /(2+a)} t^{2 /(2+a)}
$$




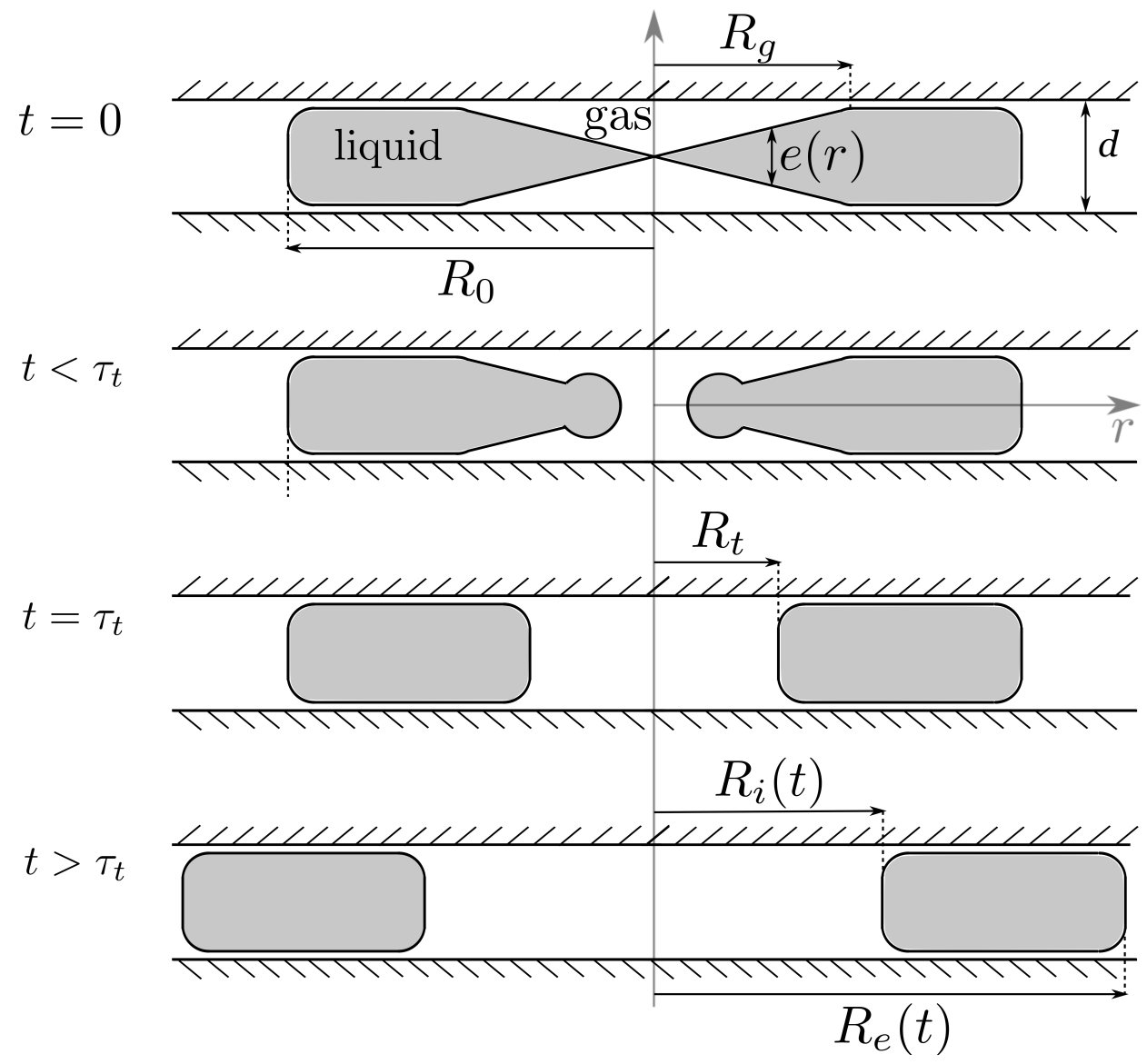

FIG. 3. Model geometry for the cross section of the confined droplets just before the hole nucleation (top), at the end of the first regime (middle) and during the second regime (bottom). In the linear region, the thickness writes $e(r)=r d / R_{g}$.

Our measurements suggest that the exponent $n=2 /(2+a)$ for the time evolution is around $2 / 3$. Consequently, it means that $a$ should be around unity and that an assuption of a linear thickness profile is reasonable. Therefore, for the case $a=1, e(r)=b_{1} r$, the solution of equation Eq. 4 reads:

$$
R_{i}(t)=\alpha t^{2 / 3}
$$

In the following we note $\alpha=\left(\frac{27 \gamma}{5 \rho b_{1}}\right)^{1 / 3}$ the proportionality factor. Given the geometry of the gas pocket described above $b_{1}=d / R_{g}=d / \sqrt{3} R_{t}$ and the proportionality factor reads :

$$
\alpha=\left(\frac{27 \sqrt{3}}{5} \frac{\gamma R_{t}}{\rho d}\right)^{1 / 3}
$$

We have to note that the small variation hypothesis made by Keller writes $\frac{1}{12}\left(\frac{d}{R_{t}}\right)^{2}<<1$. For our system this assumption is fully justified. We therefore expect $\alpha$ to be proportional to $R_{t}^{1 / 3}$ for a given system and a given plate spacing.

Our model is confronted to the experimental measurements in Fig. 4a. Each time evolution is fitted by enforcing $n=2 / 3$ and by finding the best $\alpha$ fitted value. The dispersion of the $R_{t}$ values for a given spacing allows to observe some trends: the fitted value of $\alpha$ increases as $R_{t}$ increases. The larger is the spacing, the smaller the values. Given our measurements, the data are compatible with a $R_{t}^{1 / 3}$ power law, even though it is difficult to discriminate from other exponents smaller than $1 / 2$. The analysis becomes clearer while plotting the value predicted by the model. These curves pass through all series of data without any free parameter. This can also be alternatively evidenced when displaying directly the fitted value of $\alpha$ versus its value predicted by the model (Eq. 6). Once again the agreement is 
satisfactory without any free parameter (Fig. 4b). Its is worth noticing that the same scaling is retrieved by Keller ${ }^{14}$ when looking for self-similar solutions of a potential flow triggered by capillarity without any further assumption. The undulations observed upstream while the hole growths (Fig. 1) could support such hypothesis as well.
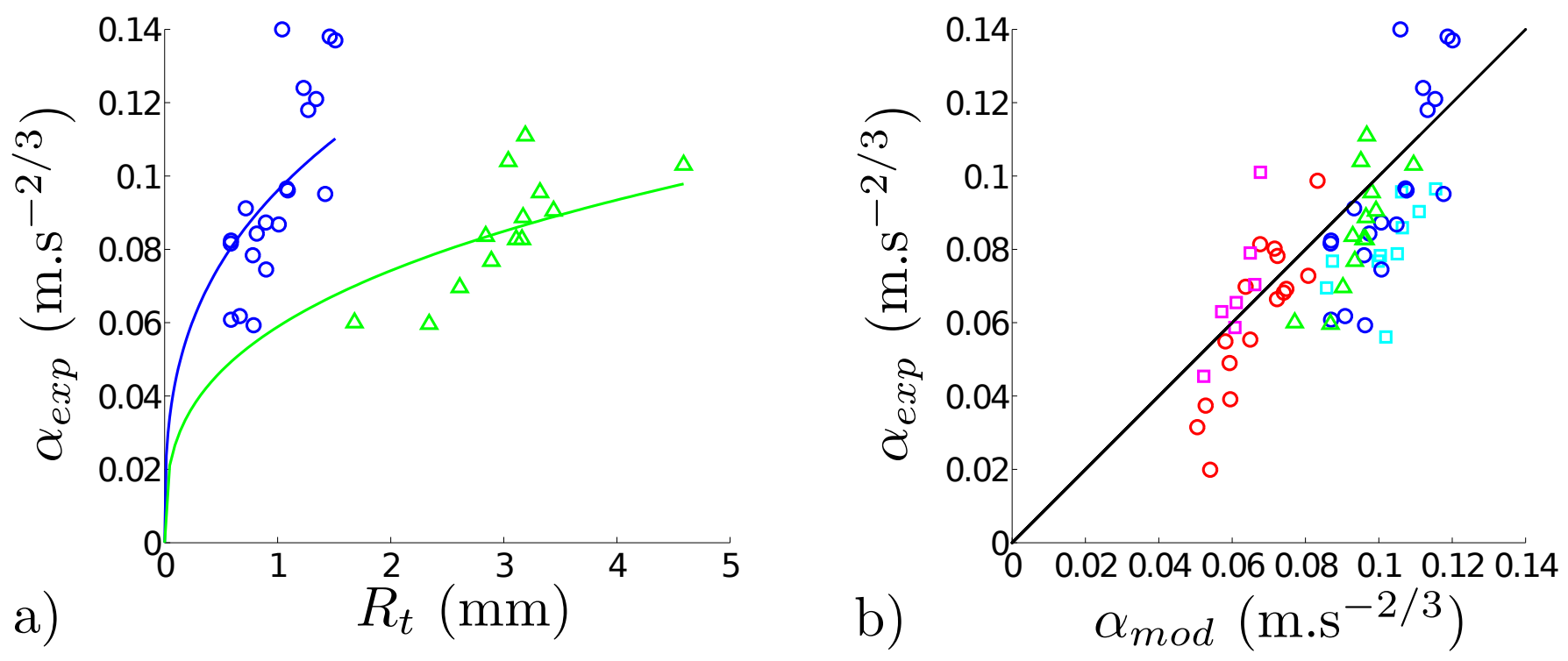

FIG. 4. Growth prefactor $\alpha$. a) experimental $\alpha$ measurements versus the transition hole radius $R_{t}$. The solid lines stand for the model predictions. For clarity, only two data sets are plotted. b) experimental $\alpha$ measurements versus the values predicted by the model. The solid line stands for the unity line and both water and nitrogen data are plotted. The following symbols are used. Water: $\circ, \square$ and $\triangle$ for $d=0.5,1$ and $2 \mathrm{~mm}$ respectively. Nitrogen: $\circ$ and $\square$ for $d=0.5$ and $1 \mathrm{~mm}$.
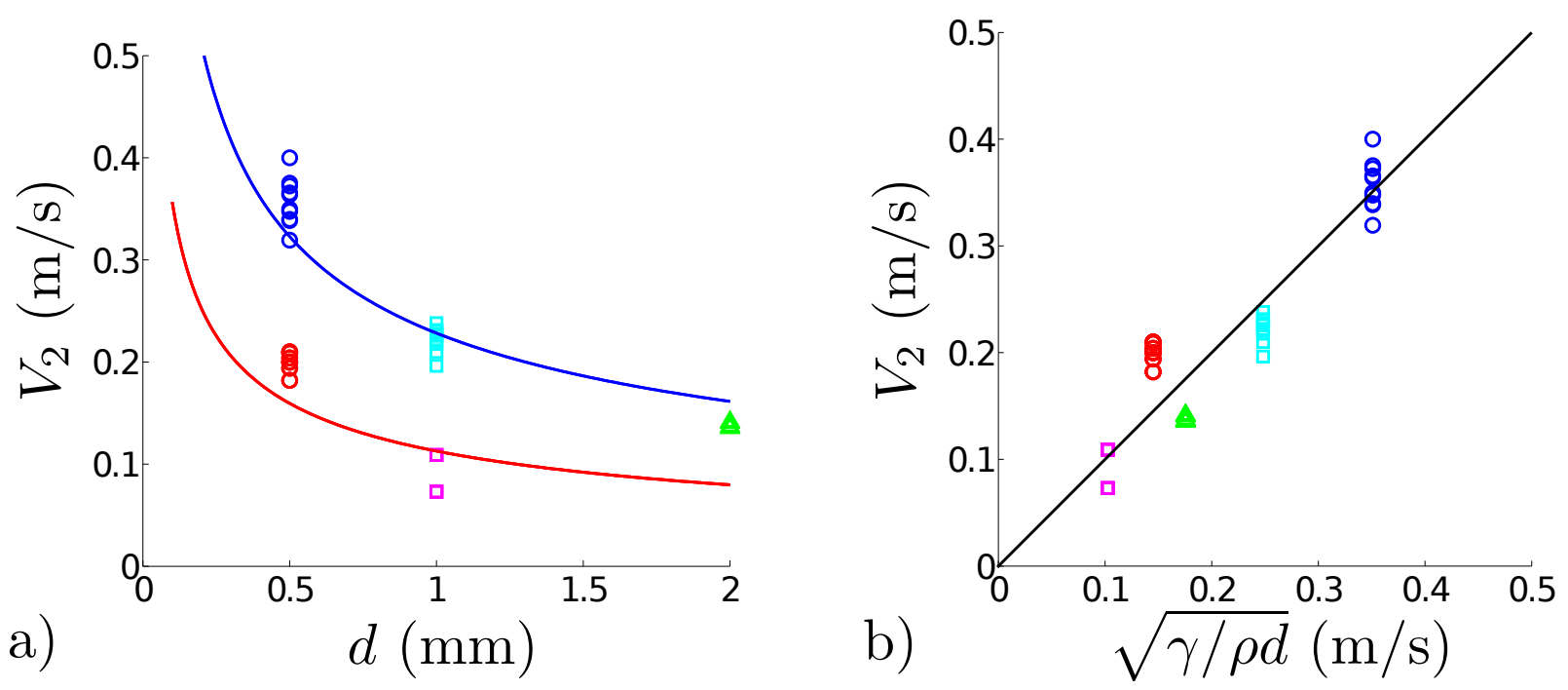

FIG. 5. Growth velocity $V_{2}$ in the second regime. a) $V_{2}$ versus $d$. Same symbols as in Fig. 4 . The solid lines are given by the equation $\sqrt{\gamma / \rho d}$ and a prefactor 0.92 for water and 1.1 for nitrogen. b) $V_{2}$ versus $\sqrt{\gamma / \rho d}$ for the same datasets. The unity line is displayed.

It is worth noticing that the development of a hole in the center of a disc-like Leidenfrost droplet has also been observed in 3D while impacting droplets on a heated surface ${ }^{15}$. In that case the mechanism is rather different: the droplets spread upon impact while partial contacts with the surface enhance the local evaporation and trigger the formation and growth of a vapor bubble in the droplets center.

We now concentrate on the second growth regime. This regime corresponds to the growth of the cylindrical gas pocket entrapped inside the liquid torus, without any liquid reorganization in the confining dimension. As can be seen in Fig. 2 the liquid equivalent radius defined above $R_{\ell}=\sqrt{R_{e}^{2}-R_{i}^{2}}$ remains constant. This point evidences the 
fact that the liquid of the torus fills all the space between the two plates except for a thin layer at the plates due to the Leidenfrost effect. While the hole grows, the torus stretches and finally breaks up when its thickness becomes comparable to the plate spacing. We show that the second regime corresponds to the inertial growth of the hole following the increase of momentum gained during the first regime. At the end of the first regime, the velocity of the hole growth writes $V_{1}=\dot{R}_{i}\left(\tau_{t}\right)=\sqrt{\frac{12}{5}} \sqrt{\frac{\gamma}{\rho d}} \simeq 1.55 \sqrt{\frac{\gamma}{\rho d}}$. The momentum at the end of the first regime is $m_{1} V_{1}$, where $m_{1}$ stands for the mass of liquid contained initially between $r=0$ and $R_{g}$ (in what follows $m_{2}$ stands for the mass of liquid initially between $r=R_{g}$ and $R_{0}$ ). This quantity is transferred to the whole liquid rim which momentum reads $\left(m_{1}+m_{2}\right) V_{2}$ at the onset of the second regime. Therefore we find by equating this two momenta that the velocity is $V_{2}=\frac{m_{1}}{m_{1}+m_{2}} V_{1}$. This reminds an inelastic collision between two liquid masses. As a consequence we expect that the velocity $V_{2}$ is constant and that its value is slightly lower than $V_{1}=\dot{R}\left(\tau_{t}\right)$ but with the same scaling. We test this assumption by plotting $V_{2}$ as a function of $d$ (Fig. 5a). Data are consistent with a $d^{-1 / 2}$ law. The scaling $\sqrt{\gamma / \rho d}$ is probed (Fig. 5b) and a linear law is obtained with a proportionality factor equals to $0.92 \pm 0.08$ for water and $1.1 \pm 0.2$ for nitrogen. Both values are slightly lower than 1.55 as expected by the model. As a remark, we mention that the torus should close if capillary forces were only are at work, the driving force being the minimization of the gas-liquid total interface.

To conclude we have studied the twofold dynamics of the hole growth in a 2D Leidenfrost droplet. Two different systems are chosen to increase the parameters range. In both cases behaviors are the same and master curves could be drawn. The first regime is reminiscent of the hole opening in micrometer-thick soap films and we have adapted the theory to the Leidenfrost case. The second regime is mostly driven by inertia and the torus expands until it fragments into droplets. A potential extension of this work would be the theoretical study of self-similar solutions for the first regime $^{14}$ adapted in the cylindrical geometry of the present study.

We would like to thank Alexandre Cohen for its careful reading of the manuscript. This study was funded by a grant from the Wolfgang Döblin Research Federation.

${ }^{1}$ J. G. Leidenfrost, De Aquae Communis Nonnullis Qualitatibus Tractatus (Duisbourg, 1756)

${ }^{2}$ A.-L. Biance, C. Clanet, and D. Quéré, "Leidenfrost drops," Phys. Fluids 15,1632 (2003).

${ }^{3}$ Y. Pomeau, M. Le Berre, F. Celestini, and T. Frisch, "The Leidenfrost effect: From quasi-spherical droplets to puddles," $C$. R. Mec. 340, 867 (2012).

${ }^{4}$ P.-G. de Gennes, F. Brochard-Wyart, and D. Quéré, Capillarity and Wetting Phenomena: Drops, Bubbles, Pearls, Waves (Springer, New York, 2003).

${ }^{5}$ J. D. Bernardin and I. Mudawar, "The Leidenfrost point: Experimental study and assessment of existing models," J. Heat Trans. 121, 894 (1999).

${ }^{6}$ H. van Dam, "Physics of nuclear reactor safety," Rep. Prog. Phys. 55, 2025 (1992).

${ }^{7}$ H. Linke, B. J. Alemán, L. D. Melling, M. J. Taormina, M. J. Francis, C. C. Dow-Hygelund, V. Narayanan, R. P. Taylor, and A. Stout, "Self-Propelled Leidenfrost Droplets," Phys. Rev. lett. 96, 154502 (2006).

${ }^{8}$ F. Celestini, T. Frisch and Y. Pomeau, "Take-off of small Leidenfrost droplets," Phys. Rev. Lett. 109, 034501(1-4) (2012).

${ }^{9}$ F. Celestini and G. Kirstetter, "Effect of the electric field on a Leidenfrost droplet," Soft Matter 8, 5992 (2012).

${ }^{10}$ K. Piroird, C. Clanet, D. Quéré, "Magnetic control of Leidenfrost drops," Phys. Rev. E 85, 056311 (2012).

${ }^{11}$ F. Celestini, T. Frisch, A. Cohen, C. Raufaste, L. Duchemin, and Y. Pomeau, "Two dimensional Leidenfrost droplets in a Hele-Shaw cell," Phys. Fluids 26, 032103 (2014).

${ }^{12}$ F. E. C. Culick, "Comments on a ruptured soap film," J. Appl. Phys. 31, 1128 (1960).

13 J. B. Keller, "Breaking of liquid films and threads," Phys. Fluids 26, 3451 (1983).

${ }^{14}$ J. B. Keller, and M. J. Miksis, "Surface tension driven flows," SIAM J. Appl. Math. 43, 268-277 (1983).

${ }^{15}$ T. Tran, H. J. J. Staat, A. Prosperetti, C. Sun, and D. Lohse, "Drop impact on superheated surfaces," Phys. Rev. lett. 108, 036101 (2012). 